\title{
ANÁLISE DE FUNCIONALIDADE DE BIOINDICADOR AMBIENTAL ATRAVÉS DE ISOTERMAS: Atherigona orientalis (DIPTERA, MUSCIDAE)
}

\author{
Vanessa da Silva Garcia \\ Doutora - Professor Departamento de Administração e Administração Pública - ICHS/UFF-VR \\ Professora Programa de Pós-Graduação em Engenharia de Produção - PPGEP/UFF-VR \\ Pesquisadora Programa de Pós-Graduação em Modelagem Computacional \\ em Ciência e Tecnologia - MCCT/UFF-VR \\ $\triangle$ vanessa.sgarcia@gmail.com

\section{Roberto de Xerez} \\ Doutor - Professor Departamento de Biologia Animal \\ Universidade Federal Rural do Rio de Janeiro - UFRRJ \\ $\bowtie$ rdexerez@ufrrj.br \\ Gustavo Aveiro Lins \\ Doutorando em Meio ambiente - UERJ \\ (OUERJ/CEDERJ/SEEDUC) \\ $\triangle$ gu.lins@terra.com.br \\ Thereza Cristina Ferreira Camello \\ Doutora em Ciências Médicas - Biomédica - Microbiologista \\ Hospital Universitário Pedro Ernesto \\ UERJ/ OUERJ/ ONU/ UN-Habitat) - RJ - Brasil \\ $\triangle$ therezacamello@gmail.com \\ Josimar Ribeiro de Almeida \\ Doutor - Professor Universidade do Estado do Rio de Janeiro (UERJ) \\ Pesquisador Senior Centro de Engenharia Nuclear/USP - RJ - Brasil \\ $\bowtie$ jralmeida@usp.br
}

\begin{abstract}
Resumo:
Atherigona orientalis (Schiner) é uma espécie de muscóide amplamente encontrado em áreas tropicais e subtropicais de todas as regiões biogeográficas. Podem ser potenciais vetores de agentes etiológicos de doenças, principalmente em áreas de poucos recursos sanitários, sendo então, consideradas como problema de Saúde Pública. Os métodos de interpretação ecológica da modificação ambiental baseiam-se na propriedade indicadora de organismos e comunidades bióticas. A multiplicidade dos diversos fatores estressantes, far-se-ão necessárias interpretações objetivas que possam fornecer uma impressão geral do efeito e da intensidade da carga existente. A dificuldade dos métodos da avaliação biológica reside em se compreender e empregar praticamente essa indicação biológica, como uma avaliação. Neste artigo a hipótese é que há um limiar térmico i.e. temperatura base (Tb) para que se processe o desenvolvimento de $A$. orientalis e o aumento e/ou diminuição da população é função de sua sobrevivência, fertilidade, razão sexual e fecundidade, com limiares influenciados pelas isotermas. A adoção dessa hipótese considera que para o grupo de espécies em questão, a desidratação não é uniforme.
\end{abstract}

Palavras-chave: Atherigona orientalis; Muscóide; Isotermas; Bioindicador ambiental. 


\title{
ENVIRONMENTAL FUNCTIONALITY BIOINDICATOR ANALYSIS THROUGH ISOTHERMS: Atherigona orientalis (DIPTERA, MUSCIDAE)
}

\begin{abstract}
:
Atherigona orientalis (Schiner) is a kind of muscóide widely found in tropical and subtropical areas of all biogeographical regions. May be potential vectors of etiological agents of diseases, especially in areas with few health resources, then being considered as a public health problem. Methods of ecological interpretation of environmental modification are based on proprietary indicator organisms and biotic communities. The multiplicity of different stressors, far will be necessary objective interpretations that can provide an overall impression of the effect and intensity of existing charge. The difficulty of biological assessment methods lies in understanding and employing just this biological indication, such an assessment. In this article the hypothesis is that there is a temperature threshold ie base temperature (Tb) that takes place the development of A. orientalis and the increase and / or decrease in population is due to its survival, fertility, sex ratio and fertility, with thresholds influenced by isotherms. The adoption of this hypothesis considers that for the group of species concerned, dehydration is not uniform.
\end{abstract}

Keywords: Atherigona orientalis; Muscoid; Isotherms; Environmental bioindicator.

\section{ANÁLISIS DE FUNCIONALIDAD DE BIOINDICADOR AMBIENTAL MEDIANTE ISOTERMAS: Atherigona orientalis (DIPTERA, MUSCIDAE)}

\section{Resumen:}

Atherigona orientalis (Schiner) es una especie de muscóide ampliamente encontrado en áreas tropicales y subtropicales de todas las regiones biogeográficas. Puede ser vectores potenciales de agentes etiológicos de enfermedades, especialmente en zonas con pocos recursos de salud, luego de ser considerados como un problema de salud pública. Métodos de interpretación ecológica de modificación ambiental se basan en organismos indicadores de propiedad y las comunidades bióticas. La multiplicidad de diferentes factores de estrés, ahora será interpretaciones objetivas necesarias que pueden proporcionar una impresión general del efecto y la intensidad de carga existente. La dificultad de los métodos de evaluación biológica radica en la comprensión y el empleo sólo por esta indicación biológica, tal evaluación. En este artículo la hipótesis es que hay una temperatura decir, base de umbral de temperatura (Tb) que tiene lugar el desarrollo de A. orientalis y el aumento y / o disminución de la población se debe a su supervivencia, la fertilidad, la proporción de sexos y la fertilidad, con umbrales influenciado por isotermas. La adopción de esta hipótesis considera que para el grupo de especies de que se trate, la deshidratación no es uniforme.

Palabras clave: Atherigona orientalis; Muscóide; Isotermas; Bioindicador ambiental.

\section{INTRODUÇÃO}

Atherigona orientalis (Schiner) é uma espécie de muscóide amplamente encontrado em áreas tropicais e subtropicais de todas as regiões biogeográficas. São encontrados desde 
excrementos humanos ao exterior de várias frutas e legumes em decomposição (ANDRZEJ e PAPE, 2014; HIBBARD e OVERHOLT, 2013). Em estudos realizados no Jardim Zoológico do Rio de Janeiro, Brasil, A. orientalis apresentou poucos ovos de helmintos na superfície externa de seu corpo, e não foi observada criando-se em fezes de animais, provavelmente devido a sua preferência alimentar por matéria orgânica vegetal (OLIVEIRA, MELLO e D’ALMEIDA, 2002 ).

Os muscóideos devido à estrutura física e ao modo de vida, alimentam-se de sangue, carcaças em decomposição e fezes, carreiam no tubo digestivo, patas e cerdas, vírus, bactérias, ovos de helmintos e outros organismos. Podedo disseminar os agentes causadores da poliomielite, febre tifóide, disenteria bacilar, disenteria amebiana e infecções helmintosas (ALMEIDA e CARVALHO, 1983; ARAUJO et al., 2005; ALMEIDA e LINS, 2010). Deste modo considera-se a relevância que esses insetos assumem em Saúde Pública, como potenciais vetores de agentes etiológicos de doenças, principalmente em áreas geográficas mais quentes e, ou em locais de parcos recursos sanitários. Em zonas rurais, contribuem para a diminuição da produção de ovos, dispersão de doenças animais, além de causar incômodo à população vizinha às criações (MARCHIORI, et al., 2007; MOURA e ARANTES, 2013).

Os métodos de interpretação ecológica da modificação ambiental baseiam-se na propriedade indicadora de organismos e comunidades bióticas. Justamente, pela multiplicidade dos diversos fatores estressantes, far-se-ão necessárias interpretações objetivas que possam fornecer uma impressão geral do efeito e da intensidade da carga existente (ALMEIDA et al., 2001; ARAUJO e ALMEIDA, 2013; CARVALHO et al.,1984,1985). A dificuldade dos métodos da avaliação biológica reside em se compreender e empregar praticamente essa indicação biológica, no sentido de uma avaliação objetiva (MIZUGUCHI et al., 1982,1985). O número de tentativas de se encontrar métodos adequados e interpretações críticas sublinha a problemática de um julgamento objetivo por meio de organismos indicadores. Principalmente os sistemas que são baseados na catalogação empírica das propriedades indicadoras de espécies animais e vegetais, refletem nitidamente as restrições de um julgamento por meio de bioindicadores, cujas valências ecológicas não são suficientemente conhecidas (ALMEIDA et al., 2004).

A capacidade inata de aumentar em número depende grandemente das condições climáticas do local onde o organismo se desenvolve. Fatores físicos do tempo como a temperatura e umidade, constituem fatores dos mais importantes, atuando conjuntamente 
sobre a capacidade reprodutiva no caso de insetos. Para avaliar a sua influência, traça-se sobre um climograma integrado com as linhas de razão finita de aumento (1), calculadas para diferentes combinações de temperatura e umidade. Tais isolinhas são resultantes do desenvolvimento de tabelas de vida nas condições especificadas no climatograma. Adicionase aos dados do climatograma que fornecem indicação do local e época do ano favoráveis para reprodução e desenvolvimento do inseto, os dados de capacidade de aumento em função do local e da época do ano. Pode-se comparar também dados entre duas populações em função do local, época do ano e ainda do recurso trófico explorado (ALMEIDA, et al., 1985, 1986, MIZUGUCHI e ALMEIDA, 1983, MIZUGUCHI et al.,1985).

A constante térmica é uma equação da hipérbole retangular que relaciona temperatura com o tempo de desenvolvimento biológico, onde $\mathrm{K}=\mathrm{y}(\mathrm{t}-\mathrm{a})$. Sendo $\mathrm{K}$ a constante térmica expressa em graus dia (GD), y o tempo requerido para completar o desenvolvimento (dias), t a temperatura ambiente $\left({ }^{\circ} \mathrm{C}\right)$, a, corresponde a temperatura do limiar do desenvolvimento $\left({ }^{\circ} \mathrm{C}\right.$ ), e t - a, é a temperatura efetiva. A velocidade do desenvolvimento pode ser calculada pela sua recíproca $\mathrm{v}=(1 / \mathrm{y})$, sendo $\mathrm{y}=(\mathrm{K} / \mathrm{t}-\mathrm{a})$. Portanto $(1 / \mathrm{y})=(\mathrm{t}-\mathrm{a} / \mathrm{K})$, e logo fazendo-se $(1 /$ $\mathrm{K})=\mathrm{b}$ constante, fica: $(1 / \mathrm{y})=\mathrm{bt}-\mathrm{ab}=\mathrm{bt}-\mathrm{K}$, onde $\mathrm{K}$ é uma constante. Então $(1 / \mathrm{y})=b t+\mathrm{K}$, representa uma equação de reta. No entanto a velocidade de desenvolvimento é uma reta, apenas numa estreita faixa de temperatura, em torno do ótimo, pois atingindo os extremos adquire uma forma sigmóide (ALMEIDA et al., 1984, 1985, 1986).

Por outro lado a equação logística que é mais realista permite uma compreensão melhor desses fenômenos. Esta equação, (fórmula de Pearl-Verhulst), é expressa por $(1 / \mathrm{K})=$ $\mathrm{K} /[1+\exp (\mathrm{a}-\mathrm{bt})]$ ou $(100 / \mathrm{y})=\mathrm{K} /[1+\exp (\mathrm{a}-\mathrm{bt})]$ quando se coloca nas ordenadas a percentagem média do desenvolvimento em vez da duração do desenvolvimento. A curva de duração de desenvolvimento é dada por $\mathrm{y}=[1+\exp (\mathrm{a}-\mathrm{bt})] / \mathrm{K}$. Para estudos desta natureza, as técnicas de análise de sistemas e modelagem matemática são particularmente úteis. Dentre os modelos utilizados os mais versáteis e de melhor operacionalidade no tratamento matemático, são os determinísticos compartimentais.

Nesse trabalho a hipótese é que há um limiar térmico i.e. temperatura base (Tb) para que se processe o desenvolvimento de A. orientalis e o aumento e/ou diminuição da população é função de sua sobrevivência, fertilidade, razão sexual e fecundidade, com limiares influenciados pelas isotermas. 


\section{METODOLOGIA}

As moscas foram capturadas com um tipo de armadilha para dípteros construída com armação de metal pintado de preto, medindo $22 \mathrm{~cm}$ de diâmetro por $40 \mathrm{~cm}$ de altura com duas aberturas - tipo veneziana - de $3 \mathrm{~cm}$ de largura na parte inferior, a fim de permitir a entrada das moscas. No interior das armadilhas era colocado um funil de "nylon", com a base voltada para baixo, para direcionar os dípteros para a parte superior da armadilha. As armadilhas foram colocadas a uma altura de cerca de um metro do solo, distanciando $2 \mathrm{~m}$ umas das outras. Foram colocadas cinco armadilhas, uma para cada isca, em cada local de coleta.Foram utilizados cinco tipos de iscas (100 g) em cada local de coletas :carcaça de camundongo, vísceras de galinha,vísceras de peixe, cebola e fezes humanas (CALDAS et al., 1999, QUEIROZ et al., 1985).

Neste trabalho modelamos a variação diária, em número de indivíduos, da população de biovetores, considerando como fatores exógenos a temperatura e a umidade relativa do ar. A população desmembrada em quatro variáveis de estado representando cada um dos estágios de desenvolvimento de um muscóide: ovos (Ovipostura), larvas (Eclosão), pupas (Empupamento) e adultos (Emergência). A representação conceitual do modelo, pode ser visualizada por um diagrama de fluxos e estoques. Num diagrama de fluxos e estoques pensamos nas variáveis de estado como estoques ou reservatórios de alguma quantidade, que no caso deste modelo é expressa em número de indivíduos, e modelamos as relações entre as variáveis como se fossem fluxos que ocorrem entre os reservatórios. No diagrama, os reservatórios são representados por caixas e os fluxos por setas (ALMEIDA et al., 2001, 2005)

Em nosso modelo os estoques correspondem ao número de indivíduos em cada um dos quatro estágios de vida, e estes recebem fluxos de indivíduos oriundos dos estágios precedentes, de acordo com o processo de desenvolvimento típico da espécie e em função das condições ambientais. Obviamente, um aporte de indivíduos para um certo estágio significa uma depleção do mesmo número de indivíduos no estágio anterior, mas no caso dos ovos, o aporte se dá pela ovipostura das fêmeas adultas e portanto não está associado a depleção de indivíduos em qualquer outro estágio. Este aporte depende da fertilidade da população, que também é influenciada pelas condições prevalentes. Todos os estágios também sofrem depleções no seu quantitativo de indivíduos devido à mortalidade, que varia para cada estágio 
e de acordo, também, com as condições prevalentes (ALMEIDA et al., 2001; D'ALMEIDA e ALMEIDA , 1996; 1998; LÔMONACO e ALMEIDA E LINS, 1995 a,b).

O modelo em questão se refere a uma área estudada relativamente pequena que não possui barreiras capazes de promover um isolamento entre as populações. Assim, no âmbito deste modelo não consideramos movimentos migratórios, sendo, portanto a mortalidade, a natalidade e a estrutura etária os únicos fatores endógenos da população levados em conta no modelo.

O modelo foi desenvolvido de forma a representar o tamanho da população que ocupa as áreas sob influência das armadilhas. Este quantitativo local pode ser encarado como um indicador do tamanho da população em toda a reserva, que não pode ser usado para estimar o tamanho real da população, mas nos permite fazer estudos sobre a variação relativa dela (ALMEIDA et al., 2001).

Matematicamente, o modelo apresenta-se composto de quatro EDOs lineares com coeficientes variáveis, cada uma representando estágios do desenvolvimento. Os processos descritos na seção anterior foram formulados como termos dependentes da temperatura e humidade do ar, e incorporados nas EDOs como taxas que controlam as entradas e saídas de indivíduos de cada estágios.

Os processos de eclosão dos ovos, empupamento das larvas e emergência dos adultos foram modelados como funções lineares da temperatura e da humidade relativa:

$$
\begin{aligned}
& \text { Eclosão }=E c T(\text { temp })+E c H(\text { humid })+E c C \\
& \text { Empupamento }=E p T(\text { temp })+E p H(\text { humid })+E p C \\
& \text { Emergência }=E m T(\text { temp })+E m H(\text { humid })+E m C
\end{aligned}
$$

Onde temp é a temperatura do ar, humid é a umidade relativa do ar e $E c T, E c H, E c C$, $E p T, E p H, E p C, E m T, E m H$ e $E m C$ são parâmetros a ser estimados.

Para todos os estágios, a mortalidade foi modelada como uma função quadrática bivariada, de concavidade para cima, com o valor mínimo no ponto referente às condições ideais de temperatura e umidade do ar: 


$$
\begin{aligned}
& \text { MortOvos }=\text { OMT }(\text { Temp }-21)^{2}+\text { OMH }(\text { Humid }-80)^{2}+\text { OMC } \\
& \text { MortLarvas }=\text { LMT }(\text { Temp }-25)^{2}+\text { LMH }(\text { Humid }-80)^{2}+\text { LMC } \\
& \text { MortPupas }=\text { PMT }(\text { Temp }-27)^{2}+\text { PMH }(\text { Humid }-80)^{2}+\text { PMC } \\
& \text { MortAdultos }=\text { AMT }(\text { Temp }-25)^{2}+\text { AMH }(\text { Humid }-80)^{2}+\text { AMC }
\end{aligned}
$$

Onde OMT, OMH, OMC, LMT, LMH, LMC, PMT, PMH, PMC, AMT, $A M H$ e $A M C$ são os parâmetros que precisam ser estimados. Os valores numéricos nos termos referentes a temperatura e a umidade $(25,27$ e 80$)$ representam as condições ideais, e foram estimados através de experimentos em laboratório (ALMEIDA et al.,1996).

A exemplo da mortalidade, a fertilidade também foi modelada como uma função quadrática bivariada, só que com a concavidade voltada para baixo e atingindo o máximo nas condições ideais de temperatura e umidade, definidas da mesma maneira que na mortalidade.

$$
\text { OvosFemDia } \left.=\text { MaxOvos } *\left\{F e C-[\text { FeT(temp }-25)^{2}\right]-\left[\text { FeH }(\text { humid }-80)^{2}\right]\right\}
$$

Onde $\mathrm{FeC}, \quad \mathrm{FeT}$ e $\mathrm{FeH}$ são parâmetros a ser estimados. MaxOvos representa fertilidade máxima que a população pode alcançar, em termos de número de ovos postos por fêmea por dia, e também é um parâmetro que precisa ser estimado.

As variações no número de indivíduos em cada estágio de desenvolvimento foram modeladas por EDOs que sintetizam os efeitos da natalidade, da mortalidade e das passagens de estágios:

$$
\begin{aligned}
& d(\text { Ovos }) / d t=(\text { OvosFemDia } \cdot \text { propFem }) \text { Adultos- }(\text { MortOvos }) \text { Ovos }-(\text { Eclosão }) \text { Ovos } \\
& d(\text { Larvas }) / d t=(\text { Eclosão }) \text { Ovos }-(\text { MortLarvas }) \text { Larvas }-(\text { Empupamento }) \text { Larvas } \\
& d(\text { Pupas }) / d t=(\text { Empupamento }) \text { Larvas }-(\text { MortPupas }) \text { Pupas }-(\text { Emergência }) \text { Pupas }^{2} \\
& d(\text { Adultos }) / d t=(\text { Emergência }) \text { Pupas- }(\text { MortAdultos }) \text { Adultos }^{2}
\end{aligned}
$$


Onde Ovos, Larvas, Pupas e Adultos são as variáveis de estado, representando a quantidade de indivíduos em cada um dos estágios de desenvolvimento.

Foi montada, uma estação meteorológica, automática, cujo sistema de aquisição de dados por sensores em períodos determinados armazena as informações obtidas em fitas magnéticas, que posteriormente são analisadas por um sistema de microcomputação. Resulta num registro final de valores médios de temperatura e umidade do ar, radiação solar, radiação líquida, precipitação, velocidade do vento. Esses dados são utilizados para determinação dos valores de evaporação potencial através do entendimento que o balanço de energia para uma coluna que se estenda desde o solo até uma altura de referência acima da vegetação, onde são feitas as observações, pode ser descrito pela expressão: $\mathrm{Rn}-\mathrm{LeE}-\mathrm{H}-\mathrm{G}=\partial \mathrm{A} / \partial \mathrm{t}$. Em que , $\mathrm{Rn}=$ radiação líquida $\left(\mathrm{Watt} / \mathrm{m}^{2}\right) ; \mathrm{Le}=$ calor latente de vaporização $(\mathrm{Joule} / \mathrm{Kg}) ; \mathrm{E}=$ fluxo de vapor d'água $\left(\mathrm{Kg} / \mathrm{m}^{2} \mathrm{~s}\right)$ ); $\mathrm{H}=$ fluxo de calor sensível $\left(\mathrm{Watt} / \mathrm{m}^{2}\right) ; \mathrm{G}=$ fluxo de calor no solo $\left(\mathrm{Watt} / \mathrm{m}^{2}\right) ; \mathrm{A}=$ Armazenamento da calor na coluna $\left(\mathrm{Joule} / \mathrm{m}^{2}\right)$.

O armazenamento de calor na coluna deve ser constituído por termos que representem o armazenamento de calor latente e sensível no interior da mesma e pelo armazenamento decalor na massa da vegetação. A variação no tempo desses armazenamentos, segundo Thom (1977) é dada por: $\partial \mathrm{A} / \partial \mathrm{t}=$ $\int_{0}^{Z_{r}} \rho \mathrm{cp}(\partial \mathrm{T} / \partial \mathrm{t}) \mathrm{dz}+\int_{0}^{Z_{r}}(\rho \mathrm{cp} / \gamma)(\partial \mathrm{e} / \partial \mathrm{t}) \mathrm{dz}+\int_{0}^{Z_{r}} \rho \mathrm{v} \operatorname{Cvg}(\partial \mathrm{Tv} / \partial \mathrm{t}) \mathrm{dz}$.

A energia disponível na superfície é utilizada para manter os fluxos de calor sensível e de calor latente, $\mathrm{H}+\mathrm{LeE}$, sendo dada, portanto, como a soma dos termos $\mathrm{Rn}-\mathrm{G}-\partial \mathrm{A} / \partial \mathrm{t}$. Quando a superfície está saturada, a resistência rc torna-se nula, e o fluxo de vapor d'água ocorre a partir da superfície em seu valor máximo, para as condições existentes, denominado de evaporação potencial.

A equação que descreve a evapotranspiração de uma superfície não saturada, segundo a formulação Penman - Monteith, é LeE $=(\Delta \mathrm{R}+\rho \mathrm{Cp}(\mathrm{es}-\mathrm{e}) / \mathrm{ra}) /(\Delta+\gamma(1+\mathrm{rc} / \mathrm{ra}))$.Os símbolos que aparecem nas equações $\partial \mathrm{A} / \partial \mathrm{t}$ e LeE representam: $\rho=$ densidade do ar $(\mathrm{Kg} /$ $\left.\mathrm{m}^{3}\right) ; \rho \mathrm{v}=$ densidade da vegetação $\left(\mathrm{Kg} / \mathrm{m}^{3}\right) ; \mathrm{Cp}=$ calor específico do ar a pressão constante $\left(\right.$ Joule $\left./\left(\mathrm{Kg}{ }^{\circ} \mathrm{K}\right)\right) ; \mathrm{Cvg}=$ calor específico da vegetação $\left(\right.$ Joule $\left./\left(\mathrm{Kg}^{\circ} \mathrm{K}\right)\right) ; \mathrm{T}=$ temperatura do $\operatorname{ar}\left({ }^{\circ} \mathrm{K}\right) ; \mathrm{Tv}=$ temperatura da vegetação $\left({ }^{\circ} \mathrm{K}\right)$; es = pressão de saturação de vapor d'água no ar $\left(\right.$ Newton $\left./ \mathrm{m}^{2}\right) ; \mathrm{e}=$ pressão de vapor d'água no ar $\left(\right.$ Newton $\left./ \mathrm{m}^{2}\right) ; \Delta=\operatorname{des} / \mathrm{dT}\left(\right.$ Newton $/\left(\mathrm{m}^{2}\right.$ $\left.{ }^{\circ} \mathrm{K}\right)$ ); $\mathrm{R}=$ energia disponível na superfície $\left(\right.$ Watt $\left./ \mathrm{m}^{2}\right) ; \gamma=$ constante psicométrica (Newton / ( 
$\left.\left.\mathrm{m}^{2}{ }^{\mathrm{o}} \mathrm{K}\right)\right) ;$ ra $=$ resistência aerodinâmica ao fluxo d'água $(\mathrm{s} / \mathrm{m}) ; \mathrm{rc}=$ resistência de superfície ao fluxo de calor ( $/ \mathrm{m})$.

A resistência aerodinâmica ao fluxo de vapor d'água (fluxo de calor latente), é descrita pela equação $\mathrm{ra}=\{\ln ((\mathrm{Zr}-\mathrm{d}) / \mathrm{Zo})-\psi \mathrm{M}\}\{\ln ((\mathrm{Zr}-\mathrm{d}) / \mathrm{Zv})-\psi \mathrm{V}\} / \mathrm{k}^{2} \mathrm{u}$ onde, $\mathrm{Zr}=$ altura de referência onde são feitas as medidas $(m) ; d=$ altura de deslocamento do plano zero (m); Zo = comprimento de rugosidade da vegetação para momentum (m); Zv = comprimento de rugosidade da vegetação para o fluxo de vapor $(\mathrm{m}) ; \mathrm{k}=$ constante de von Karman $\approx 0,41$ (adimensional); $\psi \mathrm{M}, \psi \mathrm{V}=$ correções que devem sofrer os fluxos segundo as condições de estabilidade atmosférica em que eles ocorrem.

A integração da equação $\partial \mathrm{u} / \partial \mathrm{z}$ é dependente do conhecimento da vinculação entre a função de estabilidade $\phi \mathrm{M}$ e a altura z. Tem sido estabelecido experimentalmente, para diferentes condições de estabilidade, expressões que relacionam essa função à altura $\mathrm{z}$ e ao comprimento de estabilidade de Monin-Obukhov. A equação que expressa essa relação para a atmosfera instável é dada por $\phi \mathrm{M}=[1-16(\mathrm{Z}-\mathrm{d}) / \mathrm{L}]-1 / 4$. Se é verdadeira e universal essa relação, a equação $\partial \mathrm{u} / \partial \mathrm{z}$ pode ser integrada entre os níveis $(\mathrm{d}+\mathrm{Zo})-\mathrm{Zr}$, e a sua solução designando $\mathrm{x}_{0}=[1-16(\mathrm{Zo} / \mathrm{L})] 1 / 4$ e $\mathrm{x}=[1-16(\mathrm{Zr}-\mathrm{d}) / \mathrm{L}] 1 / 4$, resulta na equação: $\mathrm{u}=\mathrm{u} * / \mathrm{k}\{\ln ((\mathrm{Zr}-\mathrm{d}) / \mathrm{Zo})-\psi \mathrm{M}\}$, na qual, $\psi \mathrm{M} 1=\ln \left[\left(1+\mathrm{x}^{2}\right)(1+\mathrm{x})^{2} /(1\right.$ $\left.\left.+\mathrm{x}_{0}^{2}\right)\left(1+\mathrm{x}_{0}\right)^{2}\right]-2 \operatorname{arctg} \mathrm{x}+2 \operatorname{arc} \operatorname{tg} \mathrm{x}_{0}$. Procedimento análogo com relação aos perfis de pressão de vapor e temperatura, nas suas formas diferenciais, assumindo $\phi \mathrm{M}=\phi \mathrm{V}=[1$ - 16 ( $\mathrm{Z}-\mathrm{d}$ ) / L ] -1/2, para atmosfera instável, e integrando entre os níveis ( d + Zv ) e Zr, produz a função integral de estabilidade, $\psi \mathrm{V}_{1}=2 \ln \left[\left(1+\mathrm{x}^{2}\right) /\left(1+\mathrm{x}_{0}^{2}\right)\right]$. Para atmosfera estável essas funções tem forma como mostram as equações: $\psi \mathrm{M}_{2}=5$ ( $\mathrm{Z}-\mathrm{d}-\mathrm{Zo}$ ) / L e $\psi \mathrm{V}_{2}=5$ ( $\mathrm{Z}$ - $\mathrm{d}-\mathrm{Zv}) / \mathrm{L}$, nas quais é assumido que $\phi \mathrm{H}=\phi \mathrm{V}_{1}=\phi \mathrm{M}_{2}=[1+5(\mathrm{Z}-\mathrm{d}) / \mathrm{L}]$, (Web, 1979). Naturalmente às condições de neutralidade na atmosfera correspondem $\psi \mathrm{H}=\psi \mathrm{V}_{1}=\psi \mathrm{M}_{2}=0$.

Um dos critérios para o estabelecimento de medidas de estabilidade atmosférica, está apoiado na definição do tempo do "comprimento de estabilidade de Monin-Obukhov", L, cuja expressão é: $\mathrm{L}=\mathrm{u}^{*} 3 \rho \mathrm{Cp} \mathrm{T} / \mathrm{kg}(\mathrm{H}+0,07 \mathrm{LeE})$, sendo $\mathrm{u}^{*}$ a velocidade de fricção em m/s; $\mathrm{T}$ a temperatura do ar em ${ }^{\circ} \mathrm{K}$ e g a aceleração da gravidade em $\mathrm{m} / \mathrm{s}^{2}$. É aceito presentemente que a equação diferencial que expressa a variação da velocidade do vento com relação a altura, acima de um plano coberto por vegetação ou não, tenha a forma, $\phi \mathrm{M}=[\mathrm{u} * / \mathrm{k}(\mathrm{Z}-\mathrm{d}$ )] $\phi \mathrm{M}$, onde $\phi \mathrm{M}$ é uma função adimensional de estabilidade para momentum, que assume valores $>1$ quando a atmosfera é estável; $<1$ quando é instável e $=1$ quando é neutra. 
O procedimento para a determinação da evaporação potencial, adequada a um conjunto de observações meteorológicas, deve executar em cada interação os seguintes cálculos: 1) um valor para o comprimento de estabilidade de Monin-Obukhov L (na primeira iteração $\mathrm{L}=\infty) ; 2$ ) as funções de estabilidade $\psi \mathrm{M} \cdot \psi \mathrm{V}$ dadas pelas equações $\psi \mathrm{M}_{1}$ e $\psi \mathrm{V}_{1}$ ou $\psi \mathrm{M}_{2}$ e $\psi \mathrm{V}_{2}$, conforme condições de estabilidade indicadas pelo último valor de $\mathrm{L}$ que está sendo utilizado; 3) a velocidade de fricção $u *$ através da equação $U$; 4) a resistência aerodinâmica ra através da equação ra; 5) a evaporação potencial através da equação LeE ( rc = 0 ); 6) o fluxo de calor sensível $\mathrm{H}$, como termo desconhecido na equação $\mathrm{Rn}-\mathrm{LeE}-\mathrm{H}-\mathrm{G}$ $=\partial \mathrm{A} / \partial \mathrm{t} ;$ 7) um novo comprimento de estabilidade $\mathrm{L}$ com a utilização da equação correspondente; 8) verificar as correções sofridas pelas variáveis E, u* $\mathrm{H}$ e L; se forem pequenas o processo está encerrado; em caso contrário uma nova iteração deve ser realizada. Após a conclusão de duas iterações já se tem condições de definir os limites inferior e superior, Li e Ls, entre os quais deve estar a solução procurada; nas próximas iterações, objetivando um aceleramento do processo, o valor de $\mathrm{L}$ assumido no primeiro não corresponde mais ao último valor encontrado no sétimo item e sim ao ponto central do intervalo Li - Ls, designado por $\mathrm{L}_{0}$; subsequentemente à determinação de um novo valor para $\mathrm{L}$, a partir de $\mathrm{L}_{0}$, é possível reduzir-se o intervalo Li - Ls, fazendo-se com que um dos limites assuma agora o valor de $\mathrm{L}_{0}$, em correspondência a uma das seguintes situações: quando o novo valor de $\mathrm{L}>\mathrm{L}_{0}$ então $\mathrm{Li}=\mathrm{L}_{0}$; quando $\mathrm{L}<\mathrm{L}_{0}$ então $\mathrm{Ls}=\mathrm{L}_{0}$; após essa redução calculase o novo ponto central e executam-se as outras operações do procedimento.

Adotou-se o diagrama de FORRESTER (1976) para se esquematizar o modelo usado na dinâmica populacional de $A$. orientalis. Neste caso: $\mathrm{X}_{1}, \mathrm{X}_{2}, \mathrm{X}_{3}, \mathrm{X}_{4}$, correspondem aos compartimento de ovo, larva, pupa e adulto (fêmea), respectivamente; S (i) a sobrevivência de cada compartimento; $\mathrm{Tb}$ (i) foi a temperatura base $\left({ }^{\circ} \mathrm{C}\right)$ para cada compartimento; $\mathrm{K}$ (i) correspondeu a constante térmica (graus - dias = GD) para cada compartimento; RS a razão sexual da espécie (número de fêmea).(número de fêmea + número de macho $=0,5$ ); $\mathrm{FE}$ representou a fecundidade da espécie ( $\mathrm{n}^{\circ}$ ovos / fêmea); FF a fertilidade da espécie (\% de eclosão de larvas).

Para o cálculo das temperaturas diárias, utilizou-se dados de medida diretas. Os valores da temperatura base $(\mathrm{Tb})$ e da constante térmica $(\mathrm{K})$ foram determinados a partir dos dados de duração (D) de cada fase em função da temperatura (T), uma vez que $K=\mathrm{D}$. (T - 
$\mathrm{Tb})$ onde $\mathrm{T}-\mathrm{Tb}=$ temperatura efetiva do inseto. Escrevendo essa equação $\mathrm{K}=\mathrm{D}$. $(\mathrm{T}-\mathrm{Tb})$ na sua forma linear tem-se $1 / \mathrm{D}=(-\mathrm{Tb} / \mathrm{K})+(1 / \mathrm{K})$. $\mathrm{T}$.

\section{RESULTADOS E DISCUSSÃO}

Na tabela 1 são apresentados os valores médios mensais de temperatura, umidade específica do ar, velocidade do vento, radiação solar e radiação líquida, além dos totais de precipitação. Os totais de energia disponível são mostradas na Tabela 1, em equivalente de mm d'água. Nesses valores estão embutidas as variações do armazenamento de energia interior da floresta, conforme as parcelas explicitadas na equação $\partial \mathrm{A} / \partial \mathrm{t}$, na determinação dessas parcelas foi assumido que as variações temporais de temperatura e pressão de vapor d'água, observadas acima da floresta, representam o padrão de variação ao longo da coluna que se estende desde o solo até a altura de referência Zr (THOM, 1977); essa consideração permite uma imediata solução para as duas primeiras integrais da equação $\partial \mathrm{A} / \partial \mathrm{t}$, com relação a terceira integral, dessa mesma equação, que representa a variação do armazenamento de energia na massa da vegetação, no reconhecimento da importância de sua magnitude, mas na falta de estudos finalizados sobre essa parcela, foi assumido simplesmente que esse termo acompanhava o padrão de variação de armazenamento de energia no ar, na forma de calor sensível. Com relação ao fluxo de calor no solo, tendo em vista os baixos níveis de radiação que atingem o solo, em torno de $3 \%$ da radiação que alcança o topo da floresta a sua contribuição foi desprezada. A queda nos valores de radiação líquida para os horários chuvosos, nos dias escolhidos e como regra geral em todo o período de observações, é contrabalanceada pelos fluxos de calor sensível, e pelas taxas negativas da variação no armazenamento de energia no interior da floresta. 
Tabela 1. Valores médios mensais dos parâmetros meteorológicos.

\begin{tabular}{|c|c|c|c|c|c|c|}
\hline $\begin{array}{l}\text { Temperatura } \\
\text { Média }^{\circ} \mathbf{C}\end{array}$ & $\begin{array}{l}\text { Umidade } \\
\text { Média } \\
\text { Específica } \\
\text { (g/Kg) }\end{array}$ & $\begin{array}{c}\text { Déficit } \\
\text { Médio de } \\
\text { Saturação } \\
\text { (g/Kg) }\end{array}$ & $\begin{array}{l}\text { Velocidade } \\
\text { Média do } \\
\text { vento } \\
(\mathbf{m} / \mathbf{s})\end{array}$ & $\begin{array}{l}\text { Radiação } \\
\text { Líquida } \\
\text { Média } \\
\left(\mathbf{W} / \mathbf{m}^{2}\right)\end{array}$ & $\begin{array}{c}\text { Radiação } \\
\text { Solar } \\
\text { Média } \\
\left(W / m^{2}\right)\end{array}$ & $\begin{array}{c}\text { Precipitação } \\
\text { Média } \\
(\mathbf{m m})\end{array}$ \\
\hline 26,9 & 16,6 & 5,6 & 1,3 & 124 & 203 & 156 \\
\hline 25,8 & 17,8 & 3,1 & 1,2 & 101 & 153 & 197 \\
\hline 26,3 & 17,7 & 4,6 & 1,2 & 101 & 163 & 78 \\
\hline 24,8 & 16,9 & 2,4 & 1,3 & 97 & 132 & 385 \\
\hline 24,7 & 16,6 & 2,2 & 1,3 & 110 & 161 & 321 \\
\hline 23,8 & 16,5 & 2,3 & 1,3 & 96 & 137 & 425 \\
\hline 24,8 & 16,7 & 2,7 & 1,4 & 108 & 167 & 228 \\
\hline 24,9 & 16,9 & 3,1 & 1,3 & 91 & 158 & 267 \\
\hline 24,8 & 16,1 & 3,2 & 1,7 & 98 & 167 & 331 \\
\hline 25,6 & 16,1 & 4,7 & 1,4 & 123 & 181 & 67 \\
\hline 25,4 & 15,7 & 4,7 & 1,4 & 128 & 179 & 128 \\
\hline 25,4 & 16,9 & 4,5 & 1,4 & 125 & 171 & 157 \\
\hline
\end{tabular}

Para que se observe uma nova geração, considerou-se apenas o período de preoviposição das fêmeas obtidas. A partir dos dados, determinou-se a equação de desenvolvimento de $A$. orientalis cujo período pré-oviposição foi $1 / \mathrm{D}=-0,042+0,008$. T.

A partir destas equações determinou-se o número de gerações por ano de A. orientalis, para as isotermas de $19^{\circ} \mathrm{C}$ a $25^{\circ} \mathrm{C}$. Através do número de indivíduos do último compartimento (fêmeas em pré-ovoposição), obtidos através das simulações, em função do tempo, para cada isoterma, pôde-se estimar o número de gerações anuais condicionado a um sistema sem ganhos e sem perdas. 
Tabela 2. Taxas de evaporação real e potencial

\begin{tabular}{c|c|c|c}
\hline $\begin{array}{c}\text { Evaporação Real } \\
\text { Média } \\
\mathbf{E}(\mathbf{m m} / \mathbf{d i a})\end{array}$ & $\begin{array}{c}\text { Evaporação } \\
\text { Potencial Média } \\
\mathbf{E}_{\mathbf{p}}(\mathbf{m m} / \mathbf{d i a})\end{array}$ & $\begin{array}{c}\text { Evaporação } \\
\text { Média } \\
\text { Thom/Oliver } \\
\mathbf{E}_{\mathbf{T}}(\mathbf{m m} / \mathbf{d i a})\end{array}$ & $\begin{array}{c}\text { Energia } \\
\text { Média } \\
\text { Disponível }\end{array}$ \\
\hline 4,26 & 7,98 & 6,56 & 3,87 \\
4,74 & 7,34 & 5,58 & 3,76 \\
4,21 & 6,56 & 5,48 & 4,34 \\
3,66 & 6,13 & 5,26 & 4,44 \\
3,58 & 5,54 & 4,14 & 4,85 \\
3,75 & 6,19 & 4,74 & 4,61 \\
5,21 & 7,64 & 5,91 & 4.53 \\
5,69 & 8,26 & 6,39 & 3,93 \\
5,33 & 7,91 & 6,38 & 4,18 \\
5,38 & 6,74 & 5,94 & 3,82 \\
3,45 & 6,63 & 4,82 & 4,68 \\
\hline
\end{tabular}

As equações obtidas para as diversas fases, em sua forma linear foram para Ovo: 1 / $\mathrm{D}=-0,18+0,02$. T; para Larva: $1 / \mathrm{D}=-0,028+0,01$. T; para Pupa: $1 / \mathrm{D}=-0,069+0,01$ . T; e para Fase adulta $1 / \mathrm{D}=-0,4178+0,02$. T.

Em função da temperatura, A. orientalis pode apresentar de 3,97 a 8,51 e de 4,66 a 8,54 gerações por ano, quando obtidas através das equações de desenvolvimento e através das simulações, respectivamente, sendo que quanto mais alta a temperatura maior o número de gerações. O quadro 1 indica o número de gerações/ano de A. orientalis, para cada isoterma, determinado através das equações de desenvolvimento e das simulações. 
Quadro 1- Número de gerações por ano de A. orientalis por isotermas $\left({ }^{\circ} \mathrm{C}\right)$.

\begin{tabular}{ccc}
\hline Isotermas & \multicolumn{2}{c}{ Número de gerações/ano } \\
\hline$\left({ }^{0} \mathrm{C}\right)$ & $\mathrm{Ng}(\mathrm{a})$ & $\mathrm{Ng}(\mathrm{b})$ \\
\hline 25 & 3,97 & 4,66 \\
26 & 4,26 & 4,95 \\
27 & 5,31 & 5,61 \\
28 & 5,77 & 6,43 \\
29 & 6,51 & 6,61 \\
30 & 7,59 & 7,46 \\
31 & 8,51 & 8,54 \\
\hline
\end{tabular}

No quadro $1, N g$ (a) é calculado a partir das equações; $\mathrm{Ng}$ (b) é a área sob a curva do número de indivíduos do último compartimento (período pré-oviposição) em função do tempo, obtida através de simulações de um sistema fechado.

Quadro 2 - Razão populacional entre gerações de A. orientalis

\begin{tabular}{|c|c|c|c|}
\hline Razão & $1^{\mathbf{a}}$ GERAÇÃO & 2ª GERAÇÃO & $3^{\mathbf{a}}$ GERAÇÃ̃O \\
\hline $\mathrm{N} 2 / \mathrm{N} 1$ & 2,31 & 4,49 & 4,71 \\
\hline $\mathrm{N} 3 / \mathrm{N} 2$ & 4,54 & 2,38 & 4,54 \\
\hline N4 / N3 & 5,23 & 5,23 & 2,61 \\
\hline N5 / N4 & 5,34 & 5,34 & 5,34 \\
\hline
\end{tabular}

No quadro 2, Ni representa i-ésima geração completa da A. orientalis $(\mathrm{i}=1,2$..5). O número de gerações da $A$. orientalis varia linearmente com as isotermas e o número de gerações anuais para cada isoterma não difere estatisticamente quando calculadas através das equações de desenvolvimento ou através das simulações, indicando a adequação do modelo.

Não há diferença significativa entre os coeficientes angulares das equações de regressão relativas ao número de gerações anuais em função das isotermas (teste " $t$ "), obtidas a partir das simulações e através das equações de desenvolvimento (Quadro 3).

Pode-se portanto afirmar que os parâmetros utilizados nas equações matemáticas do modelo determinístico compartimental estão ajustadas, retratando a dinâmica populacional de A. orientalis. 
Quadro 3 - Teste de hipótese para os coeficientes angulares das retas (a) e (b).

(a)

(a)

(b) (b)

$-0,48 \mathrm{~ns}$

$-0,48 \mathrm{~ns}$

Através de análise de variância verificou-se a significância $(0,1 \%)$ para as equações de regressão relativas ao número de gerações anuais de $A$. orientalis em função das isotermas, para as duas situações estudadas, (Quadro 1). As equações de regressão obtidas para estas situações foram (a) $\mathrm{Ng} /$ ano $=-5,13 \mathrm{q}+0,48 . \mathrm{T}$ e (b) $\mathrm{Ng} /$ ano $=-4,45 \mathrm{q}+0,49 . \mathrm{T}$.

$\mathrm{Na}$ avaliação do modelo acrescentou-se uma sobrevivência de 50\% para cada fase além de uma fecundidade média de 150 ovos/fêmea. Devido a grande variabilidade nos dados de fertilidade de $A$. orientalis, estimou-se este parâmetro de maneira a se obter um aumento médio de 5 vezes na população das fêmeas em pré-oviposição. Através das simulações, o valor encontrado para a fertilidade foi de $80 \%$.

A lei de deficiência de saturação, afirma que quando os insetos morrem por dessecação de seus tecidos, a sua longevidade é inversamente proporcional a deficiência de saturação do ar, e estabelece que a evaporação d'água de um inseto é proporcional ao déficit saturação do ar, e estabelece que a evaporação d'água de um inseto é proporcional ao déficit de saturação do ar que envolve o mesmo. Embora isto ocorra em circunstâncias particulares, nem sempre uma função linear implica em proporcionalidade.

Assim, a perda de peso por evaporação do corpo do inseto (P) é uma função linear do déficit de saturação (d) logo: $\mathrm{P}=\mathrm{a}+\mathrm{K}$.d, sendo $\mathrm{K}$ uma constante correspondente à razão de difusão das moléculas, que é uma equação da linha reta, e num caso particular, quando essa reta corta a origem, $\mathrm{a}=0, \operatorname{logo} \mathrm{P}=\mathrm{K} . \mathrm{d}$

O principal erro dessa equação é considerar que toda perda de peso seja devido a evaporação, o que realmente não ocorre. Então, se considerar que a representa a perda de peso por outras formas (excreção, por exemplo), P - a será a perda por evaporação. Logo E = K . d, i. e., a evaporação é proporcional ao déficit de saturação, o que ocorre quando não há perdas por outras fontes $(\mathrm{a}=0)$. Partindo-se dessa hipótese, e, considerando-se que o inseto terá uma vida mais curta quanto mais rápido perder água do seu corpo, devido ao nível crítico que necessita ser mantido nele, a longevidade deverá ser inversamente proporcional a soma 
d'água perdida por unidade de tempo. Logicamente que também será inversamente proporcional ao déficit de saturação para cada temperatura, a qual será explicada por uma hipérbole.

Considerando então que todos os insetos morrem quando perdem uma quantidade constante de água de seu corpo $(\mathrm{K})$ e que a relação entre a perda d'água e o déficit de saturação é dada pela equação $\mathrm{P}=\mathrm{a}+\mathrm{Kd}$, tem-se que a longevidade $(\mathrm{L})$ será expressa pela equação $\mathrm{L}=\mathrm{K}_{2} \cdot(1 / \mathrm{P}) ; \mathrm{L}=\mathrm{K}_{2} \cdot\left(1 /\left(\mathrm{a}+\mathrm{K}_{1} \mathrm{~d}\right)\right]$. Se ocorrer apenas a perda por evaporação, tem-se $\mathrm{L}=\mathrm{K}_{2}$.(1/ $\left.\mathrm{K}_{1} \mathrm{~d}\right)$ e nesse caso, a relação entre $\mathrm{K}_{2} / \mathrm{K}_{1}$ será também constante $(\mathrm{K})$. Logo: $\mathrm{L}=\mathrm{K} / \mathrm{d}$.

Considera-se também que a razão de mortalidade é proporcional ao produto do déficit de saturação pelo tempo de exposição. Embora também, isto se aplique para muitos insetos, não se pode esquecer algumas causas de erro. A razão de perda d'água não é determinada só pelo déficit de saturação independente da temperatura. Para qualquer temperatura, a razão de perda d'água pode ser uma função do déficit de saturação mas não necessariamente proporcional a ela (ALMEIDA, et al.,. 1978, 1979 A,B , 1982).

\section{CONCLUSÃO}

Pelo conceito de Constante Térmica, o produto da duração do desenvolvimento pela Temperatura efetiva é constante, sendo medida em graus dia (GD). Porque esta unidade (GD) representa a somatória de temperaturas favoráveis ao desenvolvimento dos insetos durante este período, ou seja, as temperaturas estiveram acima da temperatura do limiar de desenvolvimento. Graficamente o Grau dia (GD) é dado pela área compreendida entre curva de temperatura diária e a temperatura base.

A relação que existe entre perda de água e o déficit de saturação não é a mesma que existe entre o déficit de saturação e a razão de mortalidade. Ao contrário, se uma é linear a outra pode ser sigmóide. Para se adotar essa hipótese há que se considerar que para o grupo de espécies em questão, às vezes pode ocorrer a perda de certa quantidade de água em alguns indivíduos e não para outros, ou seja, a desidratação não é uniforme. 


\section{REFERÊNCIAS BIBLIOGRÁFICAS}

ALMEIDA, J. R.; CARVALHO, C. Distribuição de Espécies de Phaenicia (Diptera) no Brasil. Arquivos da Universidade Federal Rural do Rio de Janeiro, Rio de Janeiro, v. 6, n.2, p. 165-171, 1983.

ALMEIDA, J. R.; OLIVEIRA, S. G. DE; BORGES, I. L.; D'ALMEIDA, J. M. Application of deterministic model of isothermals for population dynamics of Synthesiomyia nudiseta (Diptera, Muscidae). Revista Brasileira de Biologia (Impresso) (Cessou em 2001. Cont. ISSN 1519-6984 Brazilian Journal of Biology (Impresso)), v. 61, p. 141-145, 2001.

ALMEIDA, J. R.; AQUINO, A. R.; ARAUJO, G. H. S.; GUENA, A. M. O.; RIOS, E. S. Curva espécie/área para avaliação de recurso ambiental. Revista Brasileira de Pesquisa e Desenvolvimento, São Paulo, v. 6, p. 131-134, 2004

ALMEIDA, J. R.; AQUINO, A. R.; AGUIAR, L. A.; GUENA, A. M. O.; MAI, L. A. Modelagem de circulação de matéria em floresta tropical. Revista Brasileira de Pesquisa e Desenvolvimento, v. 7, p. 332-342, 2005.

ALMEIDA, J. R.; LINS, G. A. Os insetos indicadores de alteração e poluição ambiental: uma análise das espécies indicadoras e seu monitoramento. HOLOS Environment, v. 10, p. 238-244, 2010.

ARAuJO, G. H. S.; AGUIAR, L. A.; AlMEIDA, J. R.; SOARES, P. S. M.; TRINDADE, R. B. E. Seleção de Indicadores de estado e avaliação de sensibilidade dos sistemas naturais às ações antrópicas. Série Gestão e Planejamento Ambiental, v. 1, p. 1-26, 2005.

ARAUJO, G. P.; ALMEIDA, J. R. Utilização de Indicadores de Biodiversidade em Relatórios de Sustentabilidade de Empresas do Setor Eletrico Brasileiro. Revista Ibero-Americana de Ciências Ambientais, v. 4, p. 63-74, 2013.

CALDAS.A; ALMEIDA, J. R.; D'ALMEIDA, J. M. Family composition of Muscoidea communities in adjacent areas of secondary tropical forest and pasture field in Rio de Janeiro, Brazil. Revista Brasileira de Zoologia , São Paulo, v. 16, n.3, p. 899-904, 1999.

CARVAlho, C. J. B.; ALMEIDA, J. R.; JESUS, C. B. Dípteros Sinantrópicos de Curitiba e arredores (Paraná, Brasil). I. Muscidae. Revista Brasileira de Entomologia, São Paulo, v. 28, n.4, p. 551-560, 1984.

CARVAlHO, C. J. B.; MALKOWSKI, S. R.; ALMEIDA, J. R. Dípteros Sinantrópicos de Curitiba e arredores (Paraná - Brasil). Ii. Fanniidae e Anthomyiidae. Neotropical Entomology, São Paulo, v. 14, n.2, p. 277-288, 1985.

D'ALMEIDA, J. M.; ALMEIDA, J. R. Longevidade e Curva de Sobrevivência de Dípteros Caliptratos em Condições de Laboratório. Brazilian Journal of Biology, Rio de Janeiro, v. 56, n.3, p. 497-505, 1996.

D'ALMEIDA, J. M.; ALMEIDA, J. R. Nichos Tróficos em Dípteros Caliptrados no Rio de Janeiro. Brazilian Journal of Biology, Rio de Janeiro, v. 58, n.4, p. 563-570, 1998.

FORRESTER, J. W. Principles of systems. Cambridge, Wright Allen Press. Inc. 320p.1976

GRZYWACZA, A.; PAPE, T.. Larval morphology of A. orientalis (Schiner) (Diptera:Muscidae) -A species of sanitary and forensic importance Acta Tropica 137 174-184 (2014) Disponível em: <www.academia.edu/8841046/Larval_morphology_of_Atherigona_orientalis_Schiner_Diptera_Muscidae_a_species_of_sanitary and_forensic_importance> Acesso em: 03/12/2015

HIBBARD, K. L; OVERHOLT, W. A.. Pepper Fruit Fly A. orientalis (Schiner) (Insecta: Diptera: Muscidae) series of the Entomology and Nematology Department, Florida Cooperative Extension Service, Institute of Food and Agricultural Sciences, University of Florida. Original publication date September 2012. Revised April 2013. Disponivel em <http://edis.ifas.ufl.edu/pdffiles/IN/IN94800.pdf > Acesso em 03/12/2015

LOMÔNACO, C.; ALMEIDA, J. R. Estrutura Comunitária de Dípteros Muscóideos da Restinga de Jacarepaguá. Revista Brasileira de Entomologia , São Paulo, v. 39, n.4, p. 891-896, 1995. 
LOMÔNACO, C; ALMEIDA, J. R. Sazonalidade de uso de Recursos para Alimentação e Composição de Dípteros Muscoideos na restinga de jacarepaguá, RJ. Revista Brasileira de Entomologia, São Paulo, v. 39, n.4, p. 883-890, 1995.

MARCHIORI, C. H.; LELES, A. S.; CARVALHO, S. A.; RODRIGUES, R. F. Parasitóides De Dípteros Muscóides Coletados No Matadouro Alvorada Em Itumbiara, Sul De Goiás, Brasil Rev. Bras. Parasitol. Vet., 16, 4, 235-237 (Brazil. J. Vet. Parasitol.) (2007). Disponível em < http://www.scielo.br/pdf/rbpv/v16n4/a10v16n4.pdf > Acesso em: 03/12/2015

MIZUGUCHI, Y.; ALMEIDA, J. R.; XEREZ, R.; GONÇALVES, L. Ecologia de Drosophila (Diptera, Drosophilidae) no pico das Agulhas Negras, RJ: I Sitio de Crias. Dusenia, Viçosa, v. 13, n.1, p. 9-14, 1982.

MIZUGUCHI, Y.; CERQUEIRA, R.; ALMEIDA, J. R.; XEREZ, R. Velocidade de Cruzamento e Seleção R. em 'Drosophila Melanogaster', Meigen, 1830. Neotropical Entomology, São Paulo, v. 14, n.2, p. 217-224, 1985 .

MOURA, R. L.; ARANTES, L.C.. Principais dípteros necrófagos observados em carcaças oriundas de diferentes regiões biogeoclimáticas do Brasil. s.d. Disponível em <http://www.cpgls.pucgoias.edu.br/8mostra/Artigos/SAUDE\%20E\%20BIOLOGICAS/Principais\%20d\%C3\%A Dpteros\%20necr\%C3\%B3fagos\%20observados\%20em\%20carca\%C3\%A7as\%20oriundas\%20de\%20diferentes \%20regi\%C3\%B5es\%20biogeoclim\%C3\%A1ticas\%20do\%20Brasil.pdf> Acesso em: 03/12/2015

OLIVEIRA, V. C.; MELlO, R. P; D’ALMEIDA, J. M.. Dípteros muscóides como vetores mecânicos de ovos de helmintos em jardim zoológico, Brasil Rev Saúde Pública 36(5):614-20 2002. Disponível em <www.revistas.usp.br/rsp/article/view/31499/33384> Acesso em 03/12/2015

QUEIROZ, S. M. P.; DUDAS, L.; CARVALHO, C. J. B.; ALMEIDA, J. R. Bionomia de 'Sarconesia Chlorogaster' (Wiedmann, 1830) (Diptera, Calliphoridae) em Curitiba - Paraná - Brasil. Neotropical Entomology (Impresso), São Paulo, v. 14, n.1, p. 105-110, 1985.

THOM, A. S.; OLIVER, H. R. . On Penaman's equation for estimating regional evapoation. Quarterly Journal Royal Metereological Society, 103: 345-357.1977 